Sharif University of Technology
Scientia Iranica
SCIENTIA
I RAN ICA
http://scientiairanica.sharif.edu

Invited Paper

\title{
Instantaneous and equilibrium responses of the brain tissue by stress relaxation and quasi-linear viscoelasticity theory
}

\author{
M. Hosseini-Farid ${ }^{a}$, A. Rezaei ${ }^{b}$, A. Eslaminejad ${ }^{a}$, M. Ramzanpour ${ }^{a}$, \\ M. Ziejewski ${ }^{a}$, and G. Karami ${ }^{a, *}$
}

a. Department of Mechanical Engineering, North Dakota State University, Fargo, ND 58108-6050, USA.

b. Department of Physiology and Biomedical Engineering, Mayo Clinic, 200 First Street, S.W., Rochester, MN 55905, USA.

Received 24 November 2018; received in revised form 21 February 2019; accepted 4 March 2019

\author{
KEYWORDS \\ Quasi-linear \\ viscoelasticity theory; \\ Brain response; \\ Brainstem tissue; \\ Stress relaxation test; \\ Instantaneous \\ response; \\ Long-term stress.
}

\begin{abstract}
Human brain and brainstem tissues have viscoelastic characteristics and their behaviors are functions of strains as well as strain rates. Determination of the equilibrium and instantaneous stresses happening at low and high strain rates provides insights into a better understanding of the behavior of such tissues. In this manuscript, we present the results of a series of stress relaxation tests at 6 different values of strains conducted on porcine brainstem tissue samples to indirectly measure the equilibrium and instantaneous stresses. The equilibrium stresses at low strain rates were measured from long-term responses to the stress relaxation test. The instantaneous stresses at high strain rates were determined using Quasi-Linear Viscoelasticity (QLV) theory at 6 strains. The results showed that the instantaneous stresses were much larger (almost 11 times) than the equilibrium stresses across all the strains. It was concluded that the instantaneous response could reasonably be estimated from the long-term response, which could easily be measured in an experimental manner. The experimental results also showed that the reduced relaxation moduli, estimated by the QLV theory, varied for the 6 strains tested.

(C) 2019 Sharif University of Technology. All rights reserved.
\end{abstract}

\section{Introduction}

Under an impact load on the head, the brain tissue experiences diverse mechanical stresses. A Traumatic Brain Injury (TBI) may occur when induced stresses exceed the tolerance limits. In most biomechanical studies on human concussions, the attempts are focused on relating the size of the mechanical assault to the localized tissue deformation, pressure, and stressstrain response in order to diagnose the brain injury [1-

\footnotetext{
*. Corresponding author. Tel.: +1701 12315859
} E-mail address: g.karami@ndsu.edu (G. Karami)

doi: $10.24200 /$ sci. 2019.21314
6]. In such efforts, accurate measurement of generated stresses in the brain tissue is necessary for predicting the size of the injuries. It is known that brain tissue has both elastic and viscous behaviors [7-10]. A great deal of effort has been devoted, therefore, to describing material properties of the brain tissue in terms of linear viscoelastic behavior [11-13]. Quasi-linear and nonlinear viscoelastic theories have also been developed for large deformations [14-16]. The majority of these studies often characterize the tissue under simple compression, tension, or shear tests [17-21]. In some studies, the brain is characterized by simultaneously measuring the elastic and viscous responses [22,23]. In some others, only the elastic characteristics are addressed to identify the tissue response [24-26]. 


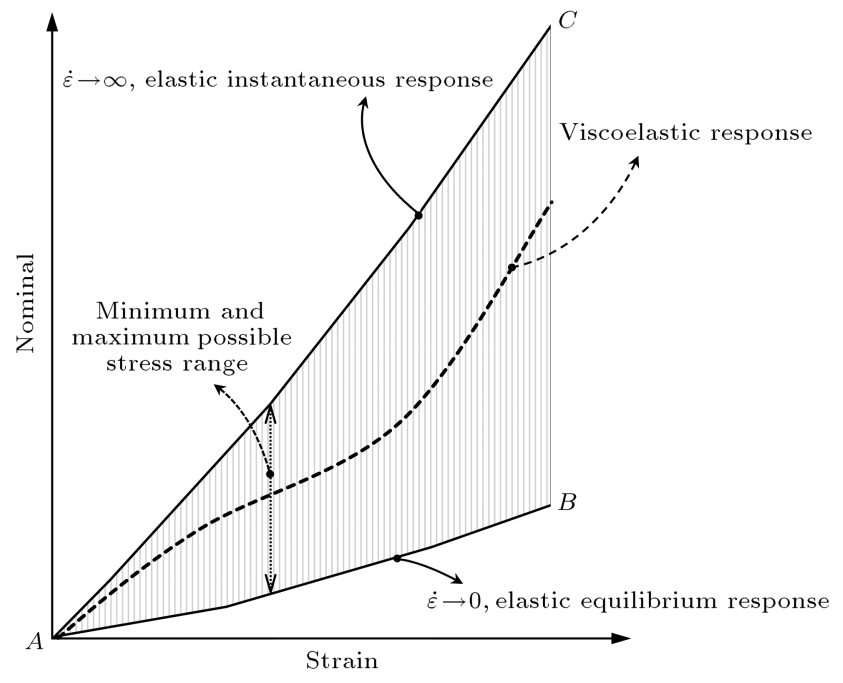

Figure 1. A schematic of possible range of stress-strain curve for viscoelastic material (brain).

Similar to soft biological tissues and other viscoelastic materials, brain tissue has a strong strain rate dependency $[27,28]$. It is also known that brain response stiffens with an increase in strain rates [29,30]. As shown in Figure 1, the stress-strain curve of a viscoelastic material, at infinitely slow rate loadings, takes the A-B path known as the equilibrium response. At infinitely high rate loadings, such as an impact or blast, the response follows the A-C path known as the instantaneous response [30,31]. Both equilibrium and instantaneous responses are purely elastic and obtainable only at extremely low or high strain rates. Such responses cannot be achieved using commercial testing machines for simple monotonic ramp experiments [32]. The viscosity domain is bounded by these two elastic responses and directly related to the material viscosity and strain rate. The maximum and minimum stress levels that can possibly be applied under a specific compression level to brain and brainstem tissues have not yet been clearly addressed.

The main aim of the current study is to estimate the instantaneous and equilibrium stresses for brainstem tissue. To this end, 6 sets of stress relaxation experiments were conducted at 6 different strain amplitudes. For these series of tests, long-term responses were measured directly by the experiments as the equilibrium stresses of the tissue. Instantaneous stresses, defined as the highest induced stresses in the tissue, were estimated by employing the QLV constitutive model [14,33].

\section{Materials and methods}

\subsection{Sample preparation}

Twelve fresh porcine brains were acquired from the Animal Science Department facilities at North Dakota
State University (NDSU). The animals were 6 to 8 months old, and all were healthy. The brains were carefully removed from the heads and cut along their mid-sagittal planes. They were then placed immediately in a physiological saline solution to prevent dehydration and kept cold while they were transported to the lab. The brains were kept at 3 to $5^{\circ} \mathrm{C}$, and all tests were conducted within 3 to 10 hours, which were the post mortem times proposed by Garo et al. [34]. Samples were prepared from both the brain and brainstem. Samples from brain tissue were tested only to validate the experimental method. Samples from brainstem tissue were then tested for the purpose of finding the instantaneous and long-term elastic responses of the tissue.

First, the samples from the brain were cut from frontal and parietal lobes of each hemisphere. The procedure followed the experimental protocol by Miller and Chinzei [15] designed to prepare samples for uniaxial unconfined compression tests. Similar to that study, a steel pipe with sharp edges and surgical scalpels were used to cut cylindrical brain samples into pieces with a diameter of $25.4 \mathrm{~mm}$ and a height of 9 to $10 \mathrm{~mm}$.

Next, the brainstem samples were cut perpendicular to the axons from the medulla oblongata and from the transitional area between the medulla and the spinal cord. Considering brainstem size limitations, the samples were prepared with a height of $5 \pm 0.7 \mathrm{~mm}$ (mean $\pm \mathrm{SD}$ ). The cross-sectional areas of the brainstems varied between samples and had complex shapes that were difficult to measure. Before each individual test, photographs of the cross-sectional areas were taken, and ImageJ software version 1.48 (Wayne Rasband, National Institutes of Health, USA) was used to calculate the size of the areas of interest. The cross-sectional area for these samples was in the range of $93 \pm 30 \mathrm{~mm}^{2}$.

\subsection{Test method}

Tests were performed at room temperature $\left(\sim 22^{\circ} \mathrm{C}\right)$ using a BOSE 3200 Electroforce machine (BOSE Corporation, Bloomington MN, USA) designed for testing soft tissue materials. Two low-force load cells, rated at $2.5 \mathrm{~N}$ and $20 \mathrm{~N}$ (BOSE Corporation, Bloomington MN, USA), were utilized for the experiments on the brainstem and brain tissue samples, respectively. Figure 2 shows the brain and brainstem samples as well as the experimental setup of the experiments.

The surface of the platens was treated with a surgical lubricant (Surgilube, Fougera Pharmaceuticals Inc.) to approximate unconfined compression tests. Samples were then carefully located between the two platens, and the upper platen was cautiously moved downward until it touched the sample. The zero force and displacement were carefully found. Based on the force and displacement signals, obtained directly from 


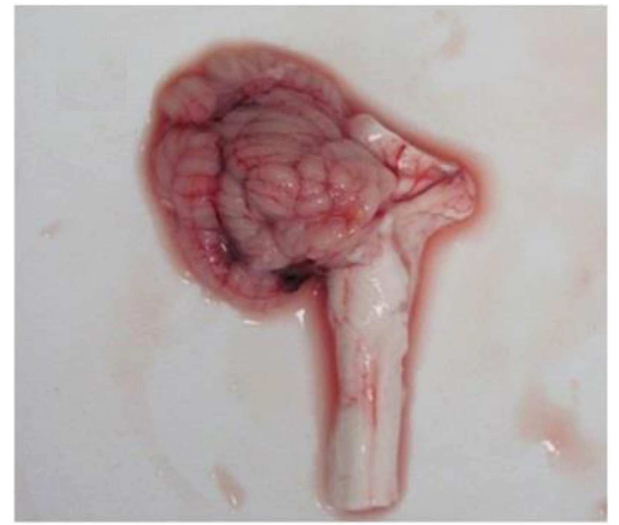

(a)

(c)

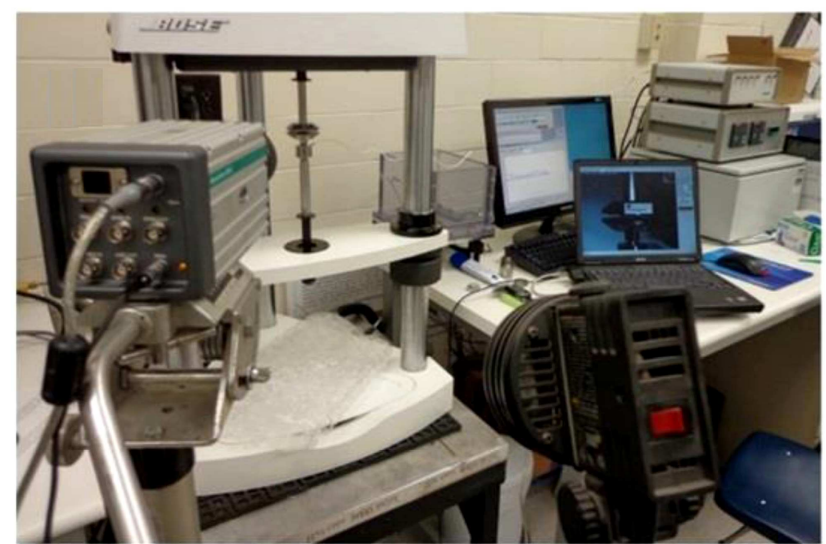

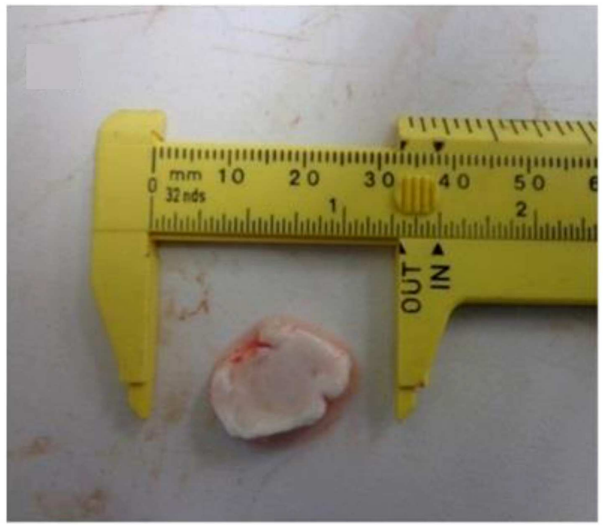

(b)

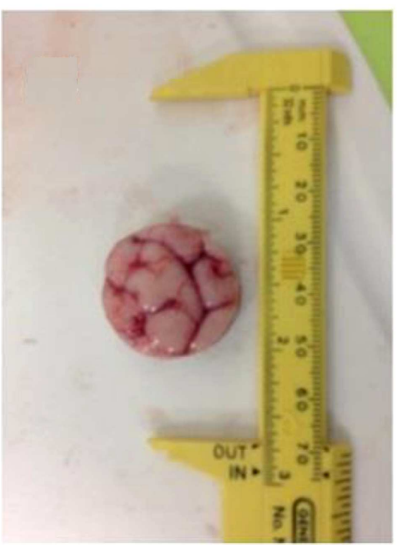

(d)

Figure 2. Sample preparation and experimental setup: (a) A porcine brainstem, (b) medulla part of the brainstem, (c) electroforce machine used for testing, and (d) a brain tissue sample.

the testing machine, nominal stresses and strains were calculated for the analyses.

For experimental validation, the brain tissue samples were tested in stress relaxation experiments at $50 \%$ initial strain ( $n=2$ samples), similar to the experiments performed by Prevost et al. [23]. Simple monotonic compression ramp tests, at strain rates of $0.64 / \mathrm{s}(n=2)$ and $0.1 / \mathrm{s}(n=3)$, were also done, resembling the studies by Miller and Chinzei [15] and Prevost et al. [23]. For brainstem tissue samples, using the same protocol for brain tissue, the stress relaxation tests were performed at 6 nominal strain amplitudes of $5 \%(n=4), 10 \%(n=3), 15 \% \quad(n=4), 20 \%$ $(n=4), 25 \%(n=4)$, and $30 \%(n=3)$. The ramp portion was set to $20 \mathrm{~mm} / \mathrm{s}$ for all of the tests, and at the end of each experiment, no signs of dehydration were observed. No notable differences were observed in mechanical properties of brainstem between different animals of the same species, even between samples of the same animal at different locations.

\subsection{Numerical formulations}

\subsubsection{Elastic responses of the tissue}

When using monotonic ramp experiments, it is very difficult to find the equilibrium stress, or the lowest strain rate, at which viscosity has no impact on the tissue response [35]. Stress relaxation tests can, however, simply explain this elastic property of the tissue by recording its behavior over a time period until the viscosity effect disappears and stress remains constant [36]. The equilibrium stress was, therefore, recorded directly from the long-term responses of each stress relaxation test. In this regard, the tissue stress values were estimated at 30 to 50 seconds after applying the initial displacement, when the measured force remained constant and the estimated stress levels did not change significantly with time. This procedure was used for all of the initial strain values to find the lower bound response of the material. Unlike the equilibrium response, for the instantaneous stress level, it is impossible directly measure the lower bound response by the stress relaxation test. Also, it is impracticable to experimentally implement a very sudden ramp. Thus, for instantaneous stresses, the QLV theory was employed to capture this elastic behavior of the tissue. The QLV mathematical model was fitted to the results of stress relaxation experiments. The fmincon solver in Matlab (Version R20102a, MathWork, MA, USA) was then 
used to find the optimal constants of the QLV model. The method provided the global minimum solution by starting from several initial values in the search domain. JMP software (Version 10.0.0, SAS Institute Inc., NC, USA) was used for statistical analyses and the significance level was set to 0.05 .

\subsubsection{Quasi-Linear Viscoelasticity (QLV) theory}

The QLV theory was employed to determine the relaxation modulus and instantaneous response of biological tissues, ranging from ligaments and tendons [37,38] to the brain $[14,39,40]$. The general QLV theory function, which incorporates both nonlinearity and time dependence of the tissue, can be expressed as:

$$
\sigma(t)=\int_{-\infty}^{t} G(t-\tau) \frac{\partial \sigma^{c}(\varepsilon)}{\partial \varepsilon} d \varepsilon(\tau),
$$

where $\sigma^{c}(\varepsilon)$ is the elastic response of the material to a suddenly applied strain, $\varepsilon ; G(t)$ is the reduced relaxation function; and $\sigma^{c}(\varepsilon)$ is assumed to be an exponential function to capture the ramp of the stress relaxation tests as follows [33]:

$$
\sigma^{c}(\varepsilon)=A\left(e^{B \varepsilon}-1\right)
$$

where $A$ and $B$ are elasticity-related tissue constants. Although for $G(t)$, a robust formula introduced in references [33,41-43], called box-shaped spectrum, can be implemented, in this study, a simpler expression is employed $[14,39]$ as the reduced relaxation function:

$$
G(t)=a e^{-b t}+c e^{-d t}+g e^{-h t}+k .
$$

In this equation, $a, b, c, d, g, h$, and $k$ are material constants $(a+c+g+k=1)$, which are estimated from the test data. According to the "strain history approach" [38], the stresses result from a ramp phase with a constant strain rate, $\dot{\varepsilon}$, over time. Thus, the subsequent stress relaxation in Eq. (1) (from $t_{0}$ to $t_{\infty}$ ) is turned into the following equation:

$$
\begin{aligned}
\sigma(t)= & A B \dot{\varepsilon}\left[\frac{a e^{-b t} e^{(b+B \dot{\varepsilon}) \tau}}{b+B \dot{\varepsilon}}+\frac{c e^{-d t} e^{(d+B \dot{\varepsilon}) \tau}}{d+B \dot{\varepsilon}}\right. \\
& \left.+\frac{g e^{-h t} e^{(h+B \dot{\varepsilon}) \tau}}{h+B \dot{\varepsilon}}+\frac{k e^{(B \dot{\varepsilon}) \tau}}{B \dot{\varepsilon}}\right] t_{0} .
\end{aligned}
$$

\section{Results}

\subsection{Experimental tests and validations}

Figure 3 shows the results of stress relaxation and simple ramp experiments on brain tissue samples along with similar results from two other previous studies by Miller and Chinzei [15] and Prevost et al. [23]. The results are very similar among studies with the RootMean-Square Deviation (RMSD) of 224 and $72 \mathrm{~Pa}$

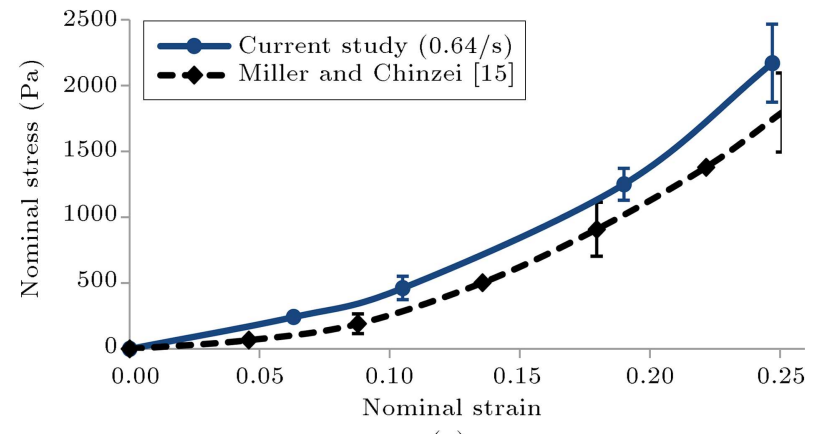

(a)

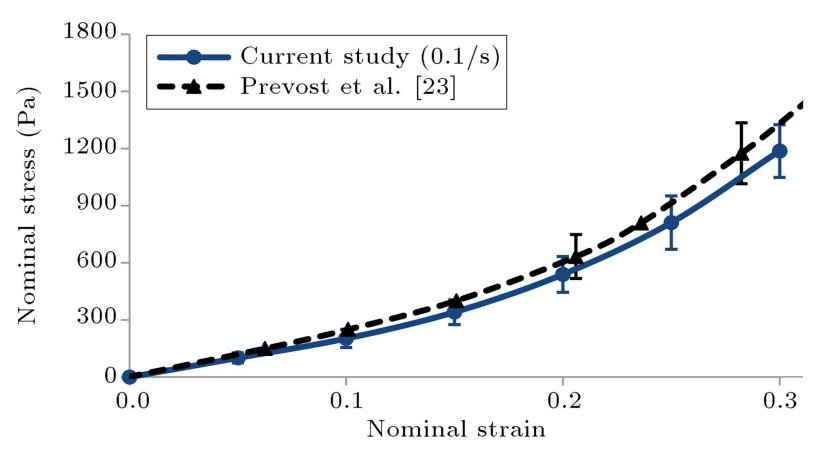

(b)

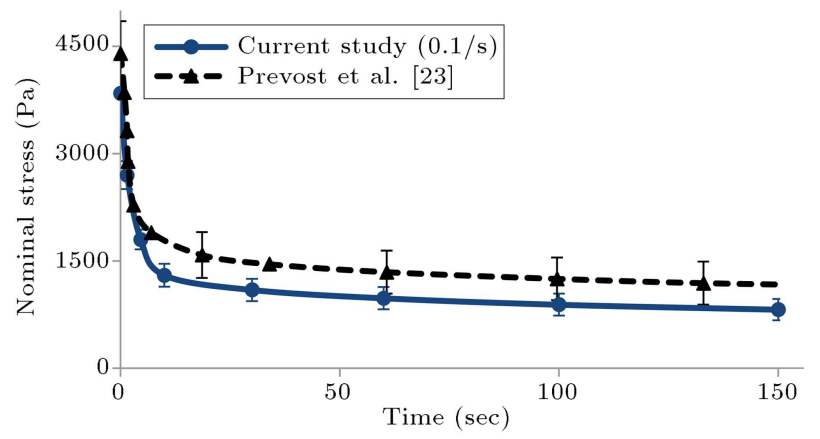

(c)

Figure 3. Results of the experimental data compared with previous studies; simple monotonic compression tests: (a) At the rate of $0.64 / \mathrm{s}$, (b) at the rate of $0.1 / \mathrm{s}$, and (c) stress relaxation at $50 \%$ strain level.

in ramp test (Figure $3(\mathrm{a})$ and (b)) and $349 \mathrm{~Pa}$ in stress relaxation (Figure $3(\mathrm{c})$ ). The results of nominal (Lagrange) stresses on the brainstem samples were averaged at each strain level as shown in Figure 4. The initial maximum stresses from stress relaxation tests varied from about $400 \mathrm{kPa}$ at $5 \%$ strain value to $3600 \mathrm{kPa}$ at $30 \%$ strain value.

The long-term stress values for all strain amplitudes were determined by the experiment presented here and shown in Figure 5. The constants of the QLV model in Eq. (4) are shown in Table 1. A significant and negative association between constant $\mathrm{B}$ and all strain values $(P=0.005)$ was found. The association for constant $A$ was insignificant $(P=0.25)$. The remaining constants did not show any associations with strain. The QLV model was then independently used to 

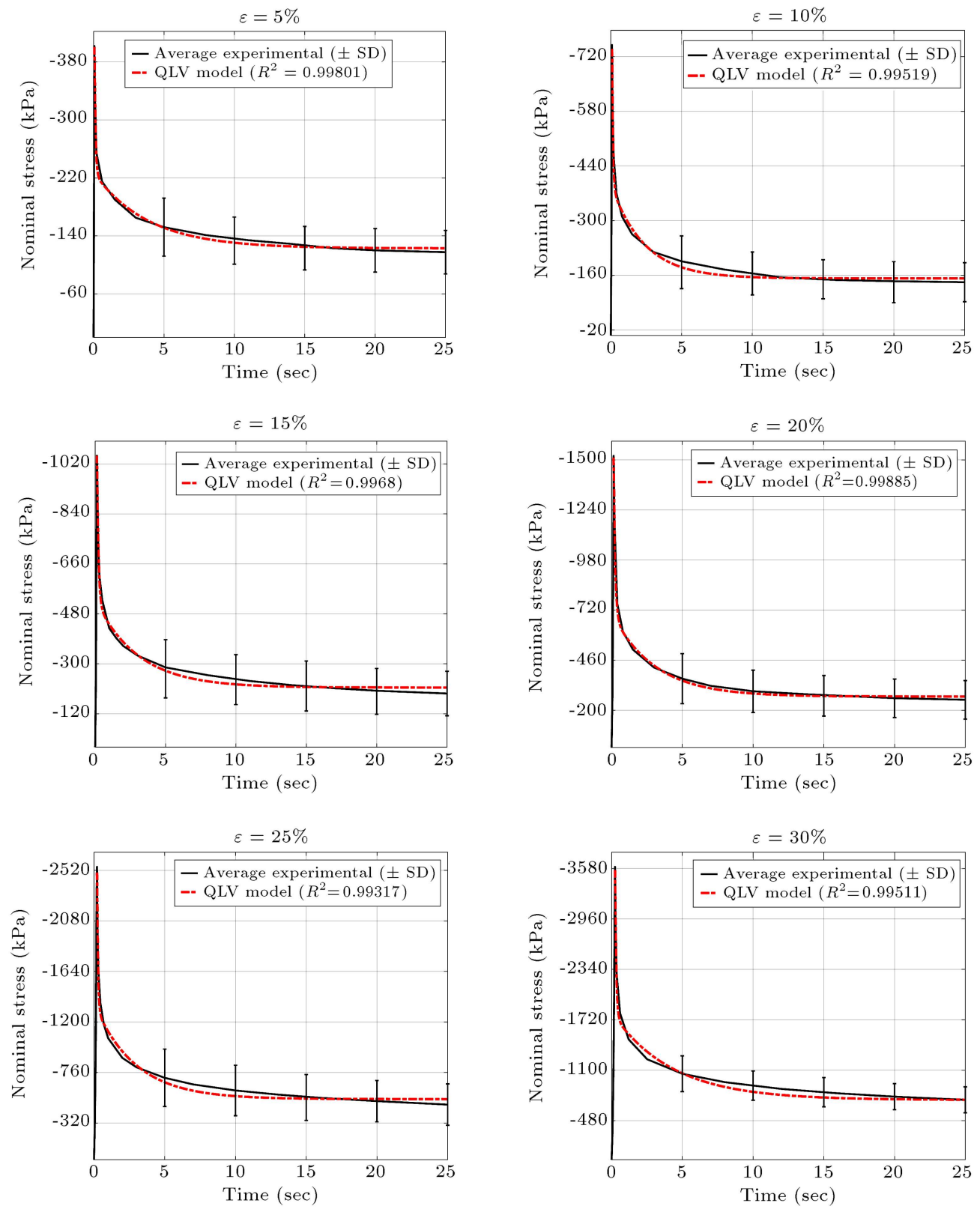

Figure 4. Six stress relaxation results of brainstem samples at strains from $5 \%$ to $30 \%$ with SDs.

Table 1. Results of QLV theory for stress relaxation.

\begin{tabular}{ccccccc}
\hline \multirow{2}{*}{$\begin{array}{c}\text { QLV } \\
\text { constant }\end{array}$} & $\mathbf{5}$ & $\mathbf{1 0}$ & $\mathbf{1 5}$ & $\mathbf{2 0}$ & $\mathbf{2 5}$ & $\mathbf{3 0}$ \\
\cline { 2 - 7 } & $\mathbf{5}$ & 387.26 & 30.629 & 587.6 & 244.5 & 481.1 \\
$B$ & 32.297 & 0.030932 & 4.6637 & 1.2916 & 0.83726 & 0.42227 \\
$a$ & 4.0902 & -300 & 23.426 & 9.0109 & 49.19 & -9.5235 \\
$b$ & 1009.7 & 14.083 & 422.48 & 1291.1 & 716.37 & 0.2387 \\
$c$ & -1.6326 & 489.66 & -13.989 & -5.4092 & -35.532 & 64.994 \\
$d$ & 13.412 & 3198 & 9.3481 & 8.9167 & 14.566 & 2908.1 \\
$g$ & -0.66758 & -114.8 & -4.9869 & -1.5865 & -7.6335 & -47.797 \\
$h$ & 0.2603 & 0.4262 & 0.33023 & 0.33128 & 0.3472 & 11.661 \\
$k$ & -0.79008 & -73.86 & -3.4501 & -1.0152 & -5.025 & -6.6729 \\
$R^{2}$ & 0.998 & 0.995 & 0.997 & 0.999 & 0.993 & 0.995 \\
\hline
\end{tabular}




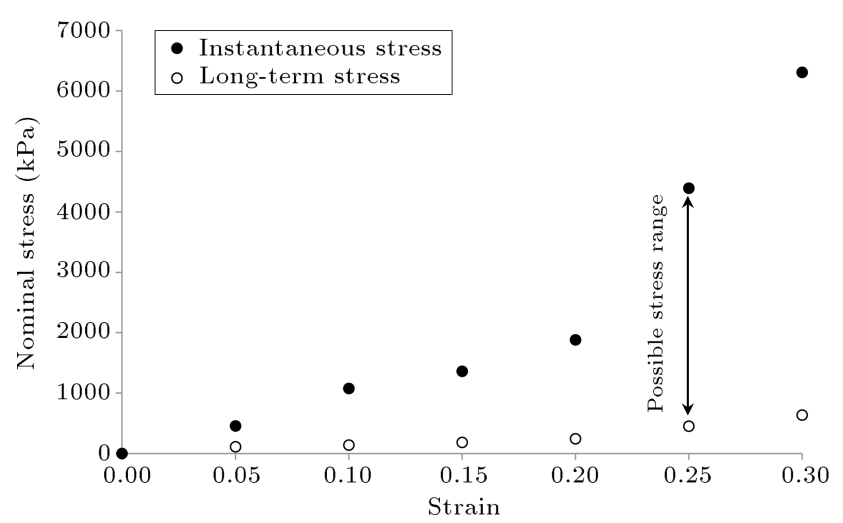

Figure 5. Instantaneous and equilibrium (long-term) stress states limiting the possible range of stress that the brainstem may experience at a specific strain value.

estimate the corresponding instantaneous stress value. In this regard, the instantaneous stress of the brainstem was calculated at each strain level by Eq. (4) when $t=0^{+}$[i.e., $G\left(0^{+}\right)=1$, such that $\sigma\left(0^{+}\right)=\sigma^{c}(\varepsilon)$ ], as shown in Figure 5. As the strain increased, the instant response also increased, as illustrated in Figure 5.

Based on a linear regression analysis, a strong linear correlation was found between the instantaneous and equilibrium stresses $(P<0.001)$. The relationship was captured by the linear equation $y=a x-b$, where $y$ and $x$ were instantaneous and long-term stresses, respectively. The constants were estimated with $95 \%$ Confidence Interval (CI) as follows: $a$ (as the slope of the regression line) $=11.0(10.2,11.9)$ and $b=$ 666.36 (365.1, 967.6). Both elastic stress values for bovine brain tissue were also derived from the data published in the study of Laksari et al. [39] and a similar regression analysis was performed. As in our porcine brainstem tissue, the resulting relationship between the two elastic responses was linear with instantaneous stresses. The determined corresponding constants were, however, different at $a=6.4$ and $b=274.27$ (Figure 6).

We also determined the reduced relaxation moduli, $G(t)$, of the brainstem at different strains. Figure 7 shows how the time dependency effect of the tissue behaved at the first 10 seconds of the test time interval, during which the responses varied considerably. As illustrated, the relaxation modulus varied from one strain level to another. The relaxation moduli derived, however, at $15 \%, 20 \%$, and $25 \%$ strain values were, to some extent, similar to each other.

\section{Discussion}

Using stress relaxation experiments, the lowest stress bound at the infinitely slow strain rates and the highest stress extent at the maximum possible strain rates on brainstem tissue were determined. Theoretically, these two boundaries should have completely elastic

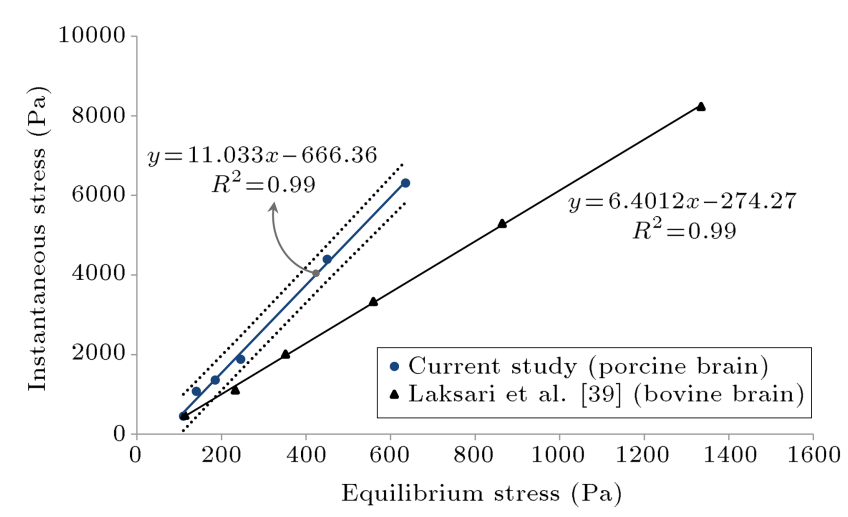

Figure 6. Correlation between instantaneous and equilibrium stresses of the brainstem starting at $5 \%$ strain level and the CI for our porcine brainstem; similar regression lines for bovine data derived from previous studies are also presented.

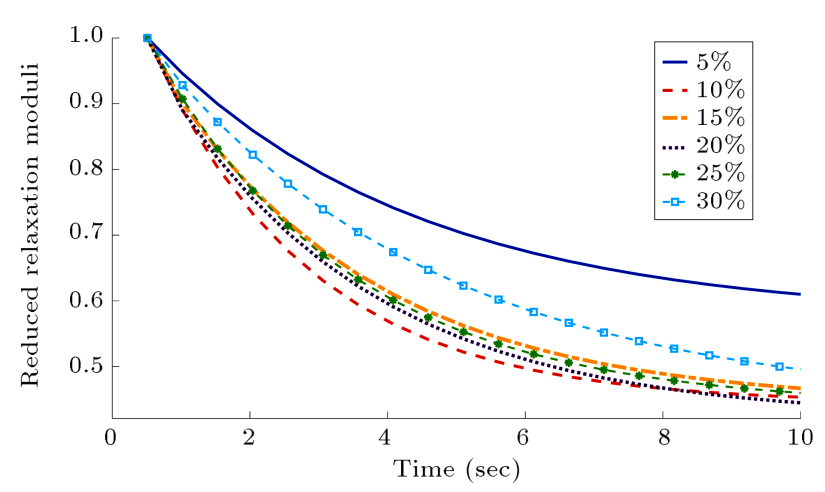

Figure 7. Reduced relaxation moduli at different strain levels for brainstem.

responses and confine the viscoelastic behavior of a very soft biological tissue. In this study, the effect of tissue viscosity was minimized to indirectly estimate these two elastic responses. Based on a strong linear correlation, it was found that the highest level of stress that could possibly be applied to the brainstem was 11 times greater than its lowest possible stress value at any tested strain amplitude. It was also found that characterization of the brain tissue, using the QLV theory at only one strain amplitude, as performed in the literature [13,14], might not well describe the applicability of the theory at different strain levels.

It is known that the instantaneous stress cannot be directly obtained by experiments due to technical issues such as overshoot, vibrations, and testing machine limitations [38]. A strong correlation was found, however, between the instantaneous and equilibrium responses $\left(R^{2}=0.99\right)$ suggesting that the instantaneous response could be estimated from the long-term response of the tissue. This result is noteworthy, because the long-term response can easily be measured directly by experiments. A similar strong linear correlation between the two elastic responses for the bovine brain tissue was also found 
in the literature [39]. This similarity in the linear relationships between the two elastic responses for both porcine and bovine suggests that a linear correlation might also hold between the two elastic responses for other types of brain tissues, possibly including human brain tissue. The slope of the regression line for the bovine brain tissue was, however, found to be smaller than that of the porcine brainstem tissue, which underscored the need to perform experiments with each particular brain tissue. This discrepancy in slopes might be due to the use of different tissues from different animals.

Reduced relaxation moduli for all strain amplitudes using the QLV theory were also measured in this study. Surprisingly, while the moduli should be independent of strain values, the experiments showed that they varied considerably with strain. This strain dependency of the relaxation modulus might be due to model truncation, as the viscous part of the tissue was considered to be linear. In reality, the viscous part of the tissue may be nonlinear $[13,44]$; thus, the application of the QLV theory may not be capable of representing the complexity of tissue behavior in the nonlinear viscoelastic regions. This finding suggests that the characterization of brain tissue using the QLV theory at only one strain amplitude may not be applicable for the whole range of tissue deformations, and multiple strain amplitude experiments must be performed to calculate a reasonable set of constants.

The two elastic responses have been addressed differently to propose brain tissue constitutive equations in prior studies [24]. Mendis et al. [45] assumed a strain rate of $0.08 / \mathrm{s}$ as quasi-static and neglected the effect of viscosity. The respective strain rate in the study by Miller and Chinzei [15] was investigated at the very small value of $0.64 \times 10^{-5} / \mathrm{s}$. In addition, Miller and Chinzei [15] assumed that the strain rate of $0.64 / \mathrm{s}$ was high enough to ignore the effects of the time-dependent relaxation parameters in their proposed model. Pervin and Chen [46], however, were able to increase the strain rates on brain tissue up to $3000 / \mathrm{s}$ using a modified split Hopkinson pressure bar and observed an increase in stress values, showing the effect of viscosity even at such high strain rates. In fact, the presence of viscosity makes it difficult to identify the long-term and instantaneous responses of the tissue at very low and very high strain rates by applying monotonic ramps. In contrast, several individual stress relaxation experiments were performed, in this study, at different strain amplitudes on tissue samples to address both elastic responses. The results showed that under initial strain levels larger than $20 \%$, the rate of change in both stresses increased. This may hint at the nature of tissue nonlinearity that, unfortunately, cannot be well addressed with simple monotonic ramps on the tissue. The application of the QLV theory gives a better estimation of the instantaneous response as the highest possible stress experienced by the tissue.

We could not find any studies on similar regions of the porcine brainstem to compare with our results. Good agreement was found, however, between results of this study and the results of the previous studies (Figure 3) on porcine brain tissue, validating the results for monotonic ramps and stress relaxation experiments. The largest difference was found between the results of the stress relaxation study presented here and those of Prevost et al. [23]. This difference was likely due to the fact that the samples in [23] were preloaded by about $30 \mathrm{~Pa}$, which possibly affected the estimated stress values.

The main limitation of this study was due to the fact that it could not be proven that the instantaneous stresses, measured indirectly using the QLV theory, were purely elastic. In addition, stress relaxation experiments were conducted at only one strain rate $\left(2 \mathrm{~s}^{-1}\right)$. To find the limits of the QLV theory for a wide range of strain values, similar stress relaxation experiments needed to be performed at different test speeds. Understanding of the test speed effect may contribute to a better understanding of the theory. The rateindependent hyperelastic constitutive models, such as Mooney-Rivlin and Ogden, also have this limitation. They have considerable error with loading speeds other than which they are determined for [8]. However, this deserves further investigation and it is suggested that other strain energy functions, such as MooneyRivlin or neo-Hookean relations, be examined. It is strongly suggested that the instantaneous behavior be determined by using different strain rates for acquiring the mean and standard deviations of that response. It is also possible that a unified model for determining the relaxation constants, using all 6 strain amplitudes simultaneously, may lead to a more robust estimation of relaxation constants over a large range of strains. On the other hand, the irregular geometries of the brainstem samples, varying from sample to sample, may have affected the estimated constants of the QLV model.

\section{Conclusion}

In this paper, a method to measure the instantaneous stress (at extremely high-speed loading) and the equilibrium stress (at remarkably slow loading) for the brainstem tissue at different strains is introduced. A series of stress relaxation test data was examined and analyzed. For equilibrium stress, the long-term responses of tissue were considered while the QLV model was employed to independently estimate the corresponding instantaneous stress at each strain. A strong linear correlation was found between the instantaneous and equilibrium behaviors of porcine 
brainstem tissue. Such linear trend also matched the data from previous studies on bovine brain tissue. The instantaneous stress for the brainstem tissue was found to be 11 times larger than the long-term stress, almost similar at all strain levels. Such findings could be used to improve evaluation of the stresses generated in brain tissue under a mechanical assault as well as to predict the injury. The reduced relaxation modulus was found to vary with strain, suggesting multiple strain experiments were still needed to investigate the range of applicability of the QLV theory.

\section{Acknowledgement}

Special thanks to the Animal Science Department at NDSU for providing the brain tissues. The work was carried out in accordance with North Dakota State University guidelines. G. Karami dedicates this manuscript to Professor Abolhasan Vafai, the Editorin-Chief of Scientia Iranica from 1993 to 2014 for his excellent editorial job.

\section{References}

1. Ratajczak, M., Ptak, M., Chybowski, L., Gawdzińska, K., and Bẹdziński, R. "Material and structural modeling aspects of brain tissue deformation under dynamic loads", Materials, 12(2), p. 271 (2019).

2. Eslaminejad, A., Hosseini Farid, M., Ziejewski, M., and Karami, G. "Brain tissue constitutive material models and the finite element analysis of blast-induced traumatic brain injury", Scientia Iranica, 25, pp. 31413150 (2018).

3. Khalid, G.A., Jones, M., Prabhu, R., Mason-Jones, A., Whittington, W., Bakhtiarydavijani, P., and Theobald, P. "Development of a paediatric head model for the computational analysis of head impact interactions", Int. J. Math. Comput. Phys., Electr. Comput. Eng., 11, pp. 113-116 (2017).

4. Hosseini-Farid, M., Ramzanpour, M., Eslaminejad, A., Ziejewski, M., and Karami, G. "Computational simulation of brain injury by golf ball impacts in adult and children", Biomedical Sciences Instrumentation, 54(1), pp. 369-376 (2018).

5. Ghajari, M., Hellyer, P.J., and Sharp, D.J. "Computational modelling of traumatic brain injury predicts the location of chronic traumatic encephalopathy pathology", Brain, 140(2), pp. 333-343 (2017).

6. Ramzanpour, M., Eslaminejad, A., Hosseini-Farid, M., Ziejewski, M., and Karami, G. "Comparative study of coup and contrecoup brain injury in impact induced TBI", Biomedical Sciences Instrumentation, 54(1), pp. 76-82 (2018).

7. Galford, J.E. and McElhaney, J.H. "A viscoelastic study of scalp, brain, and dura", Journal of Biomechanics, 3(2), pp. 211-221 (1970).
8. Farid, M.H., Eslaminejad, A., Ziejewski, M., and Karami, G. "A study on the effects of strain rates on characteristics of brain tissue", ASME 2017 International Mechanical Engineering Congress and Exposition, pp. V003T04A003-V003T04A003 (2017).

9. Farahmand, F. and Ahmadian, M. "A novel procedure for micromechanical characterization of white matter constituents at various strain rates", Scientia Iranica, http://dx.doi.org/10.24200/SCI.2018.50940.1928 (In Press).

10. Saboori, P. and Sadegh, A. "Material modeling of the head's subarachnoid space", Scientia Iranica, 18(6), pp. 1492-1499 (2011).

11. Hosseini-Farid, M., Ramzanpour, M., Ziejewski, M., and Karami, G. "A compressible hyper-viscoelastic material constitutive model for human brain tissue and the identification of its parameters", International Journal of Non-Linear Mechanics, 116, pp. 147-154 (2019).

12. Javid, S., Rezaei, A., and Karami, G. "A micromechanical procedure for viscoelastic characterization of the axons and ECM of the brainstem", Journal of the Mechanical Behavior of Biomedical Materials, 30, pp. 290-299 (2014).

13. Goriely, A., Geers, M.G.D., Holzapfel, G.A.W., Jayamohan, J., Jérusalem, A., Sivaloganathan, S., Squier, W., van Dommelen, J.A.W., Waters, S., and Kuhl, E. "Mechanics of the brain: perspectives, challenges, and opportunities", Biomechanics and Modeling in Mechanobiology, 14(5), pp. 931-965 (2015).

14. Tamura, A., Hayashi, S., Watanabe, I., Nagayama, K., and Matsumoto, T. "Mechanical characterization of brain tissue in high-rate compression", Journal of Biomechanical Science and Engineering, 2(3), pp. 115126 (2007).

15. Miller, K. and Chinzei, K. "Constitutive modeling of brain tissue: experiment and theory", Journal of Biomechanics, 30, pp. 1115-1121 (1997).

16. Rashid, B., Destrade, M., and Gilchrist, M.D. "Mechanical characterization of brain tissue in compression at dynamic strain rates", Journal of the Mechanical Behavior of Biomedical Materials, 10, pp. 23-38 (2012).

17. Darvish, K. and Crandall, J. "Nonlinear viscoelastic effects in oscillatory shear deformation of brain tissue", Medical Engineering \& Physics, 23(9), pp. 633-645 (2001).

18. Chatelin, S., Constantinesco, A., and Willinger, R. "Fifty years of brain tissue mechanical testing: from in vitro to in vivo investigations", Biorheology, 47(5$6)$, pp. 255-276 (2010).

19. Zhao, H., Yin, Z., Li, K., Liao, Z., Xiang, H., and Zhu, F. "Mechanical characterization of immature porcine brainstem in tension at dynamic strain rates", Medical Science Monitor Basic Research, 22, p. 6 (2016).

20. Moran, R., Smith, J.H., and García, J.J. "Fitted hyperelastic parameters for human brain tissue from 
reported tension, compression, and shear tests", Journal of Biomechanics, 47(15), pp. 3762-3766 (2014).

21. Destrade, M., Gilchrist, M., Murphy, J.G., Rashid, B., and Saccomandi, G. "Extreme softness of brain matter in simple shear", International Journal of Non-Linear Mechanics, 75, pp. 54-58 (2015).

22. El Sayed, T., Mota, A., Feraternali, F., and Ortiz, M. "A variational constitutive model for soft biological tissues", Journal of Biomechanics, 41, pp. 1458-1466 (2008).

23. Prevost, T.P., Balakrishnan, A., Suresh, S., and Socrate, S. "Biomechanics of brain tissue", Acta Biomaterialia, 7(1), pp. 83-95 (2011).

24. Kohandel, M., Sivaloganathan, S., Tenti, G., and Drake, J.M. "The constitutive properties of the brain parenchyma Part 1. Strain energy approach", Medical Engineering \& Physics, 28, pp. 449-454 (2006).

25. Voyiadjis, G.Z. and Samadi-Dooki, A. "Hyperelastic modeling of the human brain tissue: Effects of no-slip boundary condition and compressibility on the uniaxial deformation", Journal of the Mechanical Behavior of Biomedical Materials, 83, pp. 63-78 (2018).

26. Murphy, M., Mun, S., Horstemeyer, M., Baskes, M., Bakhtiary, A., LaPlaca, M.C., Gwaltney, S.R., Williams, L.N., and Prabhu, R. "Molecular dynamics simulations showing 1-palmitoyl-2-oleoylphosphatidylcholine (POPC) membrane mechanoporation damage under different strain paths", Journal of Biomolecular Structure and Dynamics, 37(5), pp. 1-14 (2018).

27. Hosseini-Farid, M., Ramzanpour, M., Ziejewski, M., and Karami, G. "Estimating the brain strain rates during traumatic brain injury", Biomedical Sciences Instrumentation, 54(1), pp. 361-368 (2018).

28. Farid, M.H., Eslaminejad, A., Ramzanpour, M., Ziejewski, M., and Karami, G. "The strain rates of the brain and skull under dynamic loading", ASME 2018 International Mechanical Engineering Congress and Exposition, pp. V003T04A067-V003T04A067 (2018).

29. Cheng, S., Clarke, E.C., and Bilston, L.E. "Rheological properties of the tissues of the central nervous system: a review", Medical Engineering \& Physics, 30(10), pp. 1318-1337 (2008).

30. Amin, A., Alam, M., and Okui, Y. "An improved hyperelasticity relation in modeling viscoelasticity response of natural and high damping rubbers in compression: experiments, parameter identification and numerical verification", Mechanics of Materials, 34(2), pp. $75-95$ (2002).

31. Huber, N. and Tsakmakis, C. "Finite deformation viscoelasticity laws", Mechanics of Materials, 32(1), pp. 1-18 (2000).
32. Sadeghnejad, S., Elyasi, N., Farahmand, F., Vossoughi, G., and Hosseini, S.M.S. "Hyperelastic modeling of sino-nasal tissue for haptic neurosurgery simulation", Scientia Iranica, http://scientiairanica. sharif.edu/article_21263.html (2019).

33. Babaei, B., Abramowitch, S.D., Elson, E.L., Thomopoulos, S., and Genin, G.M. "A discrete spectral analysis for determining quasi-linear viscoelastic properties of biological materials", Journal of The Royal Society Interface, 12(113), p. 20150707 (2015).

34. Garo, A., Hrapko, M., Van Dommelen, J.A.W., and Peters, G.W. "Towards a reliable characterisation of the mechanical behaviour of brain tissue: the effects of post-mortem time and sample preparation", Biorheology, 44(1), pp. 51-58 (2007).

35. Abbasi, A.A., Ahmadian, M.T., Alizadeh, A., and Tarighi, S. "Application of hyperelastic models in mechanical properties prediction of mouse oocyte and embryo cells at large deformations", Scientia Iranica, 25(2), pp. 700-710 (2018).

36. Budday, S., Sommer, G., Holzapfel, G., Steinmann, P., and Kuhl, E. "Viscoelastic parameter identification of human brain tissue", Journal of the Mechanical Behavior of Biomedical Materials, 74, pp. 463-476 (2017).

37. Toms, K., Dakin, G.J., Lemons, J.E., and Eberhardt, A.W. "Quasi-linear viscoelastic behavior of the human periodontal ligament", Journal of Biomechanics, 35(10), pp. 1411-1415 (2002).

38. Abramowitch, S.D. and Woo, S.L. "An improved method to analyze the stress relaxation of ligaments following a finite ramp time based on the quasilinear viscoelastic theory", Journal of Biomechanical Engineering, 126(1), pp. 92-97 (2004).

39. Laksari, K., Shafieian, M., and Darvish, K. "Constitutive model for brain tissue under finite compression", Journal of Biomechanics, 45, pp. 642-646 (2012).

40. De Rooij, R. and Kuhl, E. "Constitutive modeling of brain tissue: current perspectives", Applied Mechanics Reviews, 68(1), p. 010801 (2016).

41. Nigul, I. and Nigul, U. "On algorithms of evaluation of Fung's relaxation function parameters", Journal of Biomechanics, 20(4), pp. 343-352 (1987).

42. Rousseau, E., Sauren, A., Van Hout, M., and Van Steenhoven, A. "Elastic and viscoelastic material behaviour of fresh and glutaraldehyde-treated porcine aortic valve tissue", Journal of Biomechanics, 16(5), pp. 339-348 (1983).

43. Sauren, A. and Rousseau, E. "A concise sensitivity analysis of the quasi-linear viscoelastic model proposed by Fung", J. Biomech. Eng., 105(1), pp. 92-95 (1983).

44. Hrapko, M., van Dommelen, J.A.W., Peters, G.W.M., and Wismans, J.S.H.M. "The mechanical behaviour of brain tissue: large strain response and constitutive modeling", Biorheology, 43, pp. 623-636 (2006). 
45. Mendis, K.K., Stalnaker, R.K., and Advani, S.H. "A constitutive relationship for large deformation finite element modeling of brain tissue", Journal of Biomechanical Engineering, 117, pp. 279-285 (1995).

46. Pervin, F. and Chen, W.W. "Dynamic mechanical response of bovine gray matter and white matter brain tissues under compression", Journal of Biomechanics, 42(6), pp. 731-735 (2009).

\section{Biographies}

Mohammad Hosseini Farid is a PhD graduate in the Mechanical Engineering Department at North Dakota State University (NDSU). He received his MSc degree in Mechanical Engineering from Iran University of Science and Technology. His major research thrust is in the area of tissue engineering of the brain and study of the biomechanics of the trauma and impact. $\mathrm{He}$ is currently a postdoctoral research fellow in the Department of Physiology and Biomedical Engineering at Mayo Clinic in Rochester MN.

Asghar Rezaei received his PhD from the Department of Mechanical Engineering at North Dakota State University (NDSU) in 2015, and is currently a postdoctoral research fellow in the Department of Physiology and Biomedical Engineering at Mayo Clinic in Rochester MN.

Ashkan Eslaminejad received his PhD from the Department of Mechanical Engineering at North Dakota State University (NDSU) in 2018. He received MEng in Mechanical Engineering from University of Malaysia in Kuala Lumpur, Malaysia. His research areas of interest include experimental and theoretical vibration analysis, computational mechanics of fluid-structure interaction systems, and biomechanics of human head.

Mohammadreza Ramzanpour is a PhD candidate in the Mechanical Engineering Department at North Dakota State University (NDSU). He received his MSc degree in Mechanical Engineering from Sharif University of Technology. His major research thrust is in the area of tissue engineering of the brain and multiscale study of the biomechanics of brain tissue.

Mariusz Ziejewski is a Professor in the Department of Mechanical Engineering at the North Dakota State University. He received his $\mathrm{PhD}$ in Mechanical Engineering in 1986 from North Dakota State University. His areas of research interests include biomechanics of the trauma and impact, head/neck/brain computer modeling, and vehicle dynamics.

Ghodrat Karami is a Professor of Mechanical and Biomedical Engineering and Graduate Program Coordinator of the Mechanical Engineering Department at North Dakota State University (NDSU). He received his MSc degree in 1980 and PhD in 1984 in Mechanical Engineering from Imperial College of Science and Technology, University of London, England. Dr. Karami's research interests include multi-scale computational biomechanics, tissue engineering, constitutive modeling, composite engineering, and engineering design. 